\title{
Risk of malignancy in patients with rheumatoid arthritis after anti-tumor necrosis factor therapy: results from Korean National Health Insurance claims data
}

\author{
Seung Min Jung ${ }^{1}$, Seung-Ki Kwok² ${ }^{2}$ Ji Hyeon Ju², Yong-Beom Park ${ }^{1}$, and Sung-Hwan Park ${ }^{2}$
}

\author{
${ }^{1}$ Division of Rheumatology, \\ Department of Internal Medicine, \\ Yonsei University College of \\ Medicine, Seoul; ${ }^{2}$ Division of \\ Rheumatology, Department of \\ Internal Medicine, College of \\ Medicine, The Catholic University of \\ Korea, Seoul, Korea
}

Received: November 21, 2016 Revised : February 16, 2017 Accepted: April 30, 2017

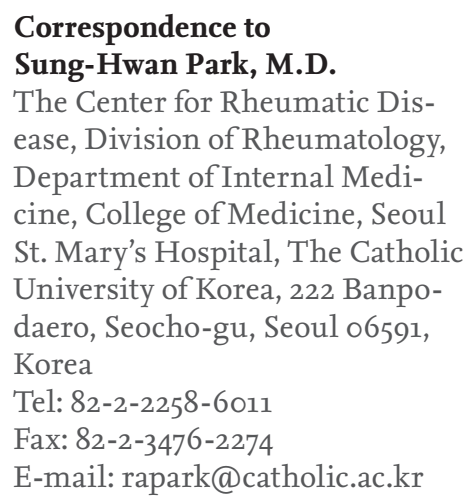

Background/Aims: Inhibition of tumor necrosis factor (TNF) is an effective treatment for rheumatoid arthritis (RA), but safety concerns about malignancy remain. The aim of this study was to evaluate cancer risk in RA patients treated with TNF inhibitors (TNFi), based on Korean Nationwide Health Insurance claims data.

Methods: Patients with seropositive RA were selected from the health insurance database containing all citizens' medical information, based on both RA diagnosis codes and medications. Between 2010 and 2014, RA patients treated with conventional synthetic disease-modifying anti-rheumatic drugs (csDMARDs) and TNFi were enrolled and followed up. We compared the cancer incidence between patients treated with TNFi and csDMARDs using incidence rate ratios (IRRs) after adjustment for age, gender, and observational periods.

Results: Of 45,423 selected patients with seropositive RA, 2,337 were treated with TNFi and 43,086 were treated with csDMARDs. The TNFi group was younger and was followed-up for a longer duration. During the observational period, 1,732 and 49 cases of cancer were detected in patients treated with csDMARDs and TNFi, respectively. Old age and male sex were associated with cancer occurrence. Adjusted IRRs for all cancers and common cancers demonstrated that cancer incidence did not differ significantly between the TNFi group and csDMARDs group (IRR $=0.913$ for all cancers, $p=0.546$ ).

Conclusions: This study revealed that cancer incidence was similar in RA patients treated with TNFi and csDMARDs. Anti-TNF therapy may be a safe therapeutic option for RA treatment, in terms of malignancy.

Keywords: Rheumatoid arthritis; Tumor necrosis factor; TNF inhibitors; Malignancy; Cancer

\section{INTRODUCTION}

Rheumatoid arthritis (RA) is a systemic autoimmune disease requiring treatment with immunosuppressive agents. Proper management of RA includes the use of disease-modifying anti-rheumatic drugs (DMARDs) to control systemic and local inflammation $[1,2]$. Recently, development of biological DMARDs, including 
tumor necrosis factor inhibitors (TNFi), has enabled more effective disease activity control. Because of the proinflammatory role of TNF, treatment with TNFi has proven to be beneficial for RA patients with inadequate responses to conventional synthetic DMARDs (csDMARDs) [3]. However, anti-TNF therapy has also raised safety concerns, because relative immune deficiency following TNF inhibition can increase the risk of infection and malignancy. Since TNF plays an important role in cancer pathogenesis, the risk for cancer during and after anti-TNF therapy has emerged as a major concern $[4,5]$.

TNF was primarily identified as an anti-tumor factor in the serum of Bacillus Calmette-Guérin-infected mice treated with endotoxin $[6,7]$. Serum transfer from these mice to other mice with subcutaneously transplanted sarcomas reduced the size of tumors. Several subsequent studies also indicated that systemic administration of TNF could suppress tumor progression, whereas other studies revealed that downregulation of TNF paradoxically reduced tumor formation and metastasis [4]. Like these preclinical studies, clinical trials were also inconclusive, with conflicting results regarding TNF effects on carcinogenesis. Both treatment with recombinant TNF and TNFi were found to be therapeutically ineffective in cancer patients [4,5].

Since TNFi has become available and widely used in RA patients, the risk for malignancy in patients treated with TNFi has been constantly studied. Earlier data from clinical trials indicated that the risk for malignancy was greater in RA patients treated with TNFi than in TNFi-naïve patients [8]. However, these studies were limited by small sample sizes and heterogeneous study populations. The majority of subsequent studies based on a large national registry suggested that overall cancer incidence did not increase during or after anti-TNF therapy [9-12].

Despite substantial previous research, efforts to elucidate the association between anti-TNF therapy and cancer are still ongoing. Because cancer incidence is influenced by genetic predisposition and environmental factors, cancer epidemiology differs between continents and countries [13]. Thus, it is desirable to estimate cancer incidence from a nationwide database. In South Korea, the national healthcare system provides insurance coverage for nearly all citizens. All hospitals and medical institutions submit insurance claims to the Health In- surance Review and Assessment Service (HIRA), which reviews all insurance claims and makes decisions regarding payment [14]. Because the submitted data include information about diagnosis and treatment for all citizens, the HIRA database can be used to obtain nationwide medical information.

In this study, we used the HIRA database to evaluate malignancy risk in RA patients following anti-TNF therapy. The incidence of all cancers and common cancers were compared in RA patients treated with TNFi or csDMARDs.

\section{METHODS}

\section{Identification of RA patients}

We retrieved data about RA patients from the HIRA database using both the diagnosis code from the International Classification of Disease, Revision 10 (ICD10) and patient medications, between January, 2010 and December, 2013. As previously reported, identification of RA patient is a critical step in studies conducted with the health insurance claims database [15,16]. The RA (Mo5) diagnostic code was primarily used to select the study population. We reviewed the lists of medications in patients with Mo5 codes to increase diagnostic reliability. Patients with RA codes were confirmed as RA patients if they had a prescription for TNFi, methotrexate, leflunomide, or more than one other csDMARD besides methotrexate or leflunomide (Fig. 1). TNFi included infliximab, etanercept, adalimumab, and golimumab. Because RA treatment with certolizumab was not covered by the National Health Insurance System during the study period, prescriptions for certolizumab were negligible. Other csDMARDs included hydroxychloroquine, sulfasalazine, cyclosporine, tacrolimus, azathioprine, mizoribine, and bucillamine. Although Mo6 codes possibly include seronegative RA, combination of Mo6 codes and medication review still showed low diagnostic reliability. Thus, patients with Mo6 codes were excluded from the analysis.

All identified patients with RA were classified according to their medications. The TNFi group was defined as patients who were prescribed with TNFi more than once. TNFi-naïve patients treated only with csDMARDs were categorized into the csDMARDs group. In the in- 
surance claims database, patient names and national registration numbers were deleted to ensure the patient confidentiality. This study was approved by the HIRA Research Ethics Committee.

\section{Malignancy definition}

Malignancy occurrence was defined as the new appearance of a cancer-related ICDio code (Coo-Dog) in the insurance claims during the study period. In these cases, previous records were reviewed to find out the past history of malignancy. If there was no reported cancer for more than 1 year prior to record acquisition, we defined that cancer as newly developed. Patients with comorbid cancer at the entry point were excluded. Since patients with cancer-related ICD1o codes were entered into a co-payment program based on the clinicopathologic evaluation, the diagnosis of cancer was quite reliable.

Follow-up duration was determined by the interval between entry into and exit from this study for each pa-

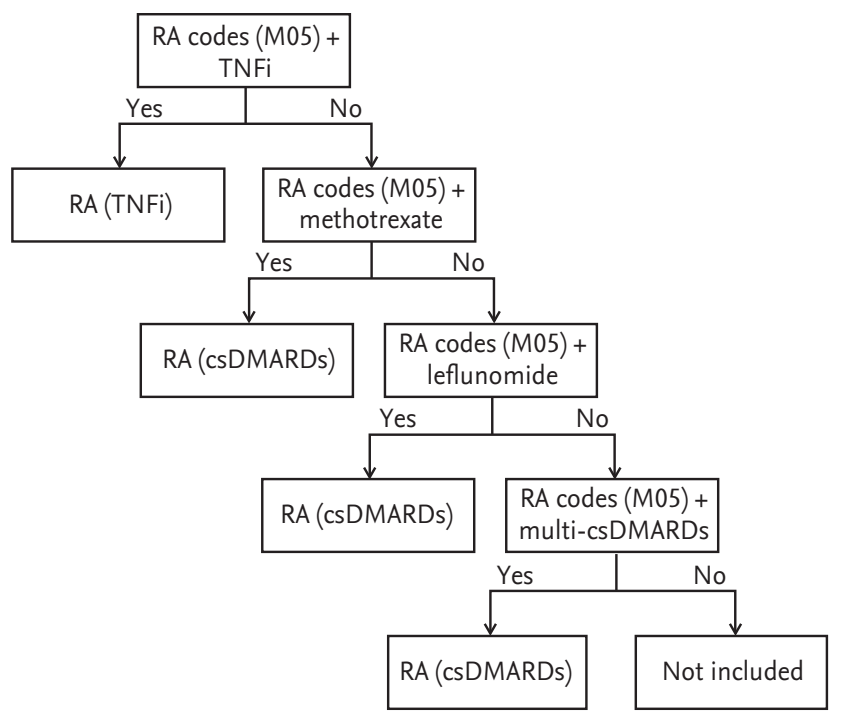

Figure 1. Selection of rheumatoid arthritis (RA) patient from the Health Insurance Review and Assessment Service (HIRA) database. Patients were classified as having RA according to their diagnosis codes and medications. If patients with RA codes (Mo5) were prescribed with tumor necrosis factor inhibitors (TNFi), methotrexate, leflunomide, or more than one conventional synthetic disease-modifying antirheumatic drugs (csDMARDs), they were classified as RA patients. Other csDMARDs except methotrexate, and leflunomide included hydroxychloroquine, sulfasalazine, cyclosporine, tacrolimus, azathioprine, mizoribine, and bucillamine. tient (Fig. 2). The entry point for patients treated with csDMARDs was RA identification. When RA codes were initially introduced within the study period, patients were enrolled at the time of RA diagnosis. For patients with new RA onset, insurance claims from the year before entry were reviewed to rule out a prior RA diagnosis. If RA codes were found before the study period, the entry point was defined as initiation of the study period. In patients treated with TNFi, the entry point was determined by TNFi treatment initiation. If a patient began TNFi treatment before 2010, the patient was traced from the study beginning. Follow-up was terminated in cases of malignancy. If a malignancy was not found, patients were traced until the end of the study.

\section{Statistical analysis}

Demographic data such as age and gender were compared with Student $t$ test and chi-square tests, respectively. Differences in time to cancer diagnosis between

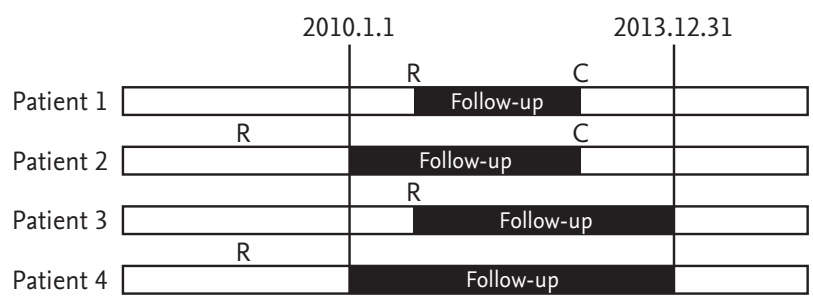

Patients treated with conventional synthetic DMARDs

A

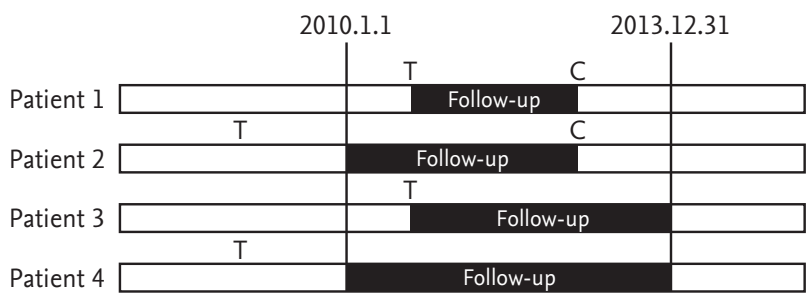

Patients treated with TNFi

\section{B}

Figure 2. Schematic diagram showing the study design. (A) Patients treated with conventional synthetic diseasemodifying anti-rheumatic drugs (csDMARDs) were enrolled at study initiation or rheumatoid arthritis (RA) diagnosis. Patients were traced until cancer occurrence or study termination. (B) Patients treated with tumor necrosis factor inhibitors (TNFi) were enrolled at introduction of anti-TNF therapy or study initiation. Follow-up was terminated when cancer was newly detected or the study period ended. R, diagnosis of RA; C, diagnosis of cancer; T, introduction of TNFi. 
patients treated with TNFi and csDMARDs were analyzed with the Wilcoxon rank-sum test.

Cancer incidence was presented as events/100,000 person-years for the entire study population. The incidence rate ratio (IRR) was estimated with a Cox regression model, adjusted for age, gender, and follow-up duration. We compared the incidence for all cancers and site-specific cancers between the TNFi and csDMARDs groups with IRR. A $p<0.05$ was considered significant. All statistical analyses were performed with SAS 9.1 software (SAS Institute Inc., Cary, NC, USA).

\section{RESULTS}

\section{Patient characteristics}

We retrieved 45,423 patients with seropositive RA from the HIRA database, based on the algorithm for patient selection (Fig. 1). Of the selected patients, 2,337 were treated with TNFi. Patient characteristics at study enrollment are presented in Table 1. The mean age of RA patients was 57.49 years, and $79.7 \%$ of patients were female. The TNFi group was younger than the csDMARDs group, but the sex ratios were similar between the two groups. More patients in the TNFi group were enrolled in 2010, and thus follow-up duration was longer for the TNFi group (3.49 years vs. 3.19 years for the csCMARDs group; $p<0.001$ ). The most frequently used drug in the TNFi group was etanercept (40.95\%), followed by adalimumab (39.97\%), infliximab (17.93\%), and golimumab (1.16\%).

\section{Characteristics of RA patients with cancer}

Among 45,423 patients, 1,781 cancer cases were newly detected. Cancer diagnosis was more frequent in elderly patients, and the proportion of male patients was higher in cancer group. Distribution of TNFi did not differ significantly between patients with and without cancer (Table 2).

During the observational period, 1,732 patients (4.02\%) in the csDMARDs group and 49 patients $(2.10 \%)$ in the TNFi group were diagnosed with cancer (Table 3). The crude incidence rates for cancer were 1,257/100,000 patient-years and 600/100,000 patient-years in patients treated with csDMARDs and TNFi, respectively. The median interval between enrollment and cancer diagnosis was 20.06 months for the entire study population, and the time to cancer diagnosis was comparable in pa-

Table 1. Baseline characteristics for Korean patients with rheumatoid arthritis

\begin{tabular}{|c|c|c|c|c|}
\hline Characteristic & All patient $(n=45,423)$ & $\begin{array}{l}\text { Patient treated with csDMARD } \\
\qquad(\mathrm{n}=43,086)\end{array}$ & $\begin{array}{l}\text { Patient treated with TNFi } \\
\qquad(\mathrm{n}=2,337)\end{array}$ & $p$ value \\
\hline Age, yr & $57.49 \pm 13.25$ & $57.70 \pm 13.17$ & $53.63 \pm 14.04$ & $<0.001$ \\
\hline Female sex & $36,212(79 \cdot 72)$ & $34,382(79.80)$ & $1,830(78.31)$ & 0.080 \\
\hline Use of MTX & $33,366(73.46)$ & $31,359(72.78)$ & $2,007(85.88)$ & $<0.001$ \\
\hline Calendar year of enrollment & & & & $<0.001$ \\
\hline 2010 & $33,432(73.60)$ & $31,546(73.22)$ & $1,886(80.70)$ & \\
\hline 2011 & $3,728(8.21)$ & $3,543(8.22)$ & $185(7.92)$ & \\
\hline 2012 & $3,959(8.72)$ & $3,805(8.83)$ & $154(6.59)$ & \\
\hline 2013 & $4,304(9.48)$ & $4,192(9 \cdot 73)$ & $112(4.79)$ & \\
\hline Duration of follow-up, yr & $3.21 \pm 1.23$ & $3.19 \pm 1.24$ & $3.49 \pm 0.99$ & $<0.001$ \\
\hline \multicolumn{5}{|l|}{ Distribution of TNFi } \\
\hline Etanercept & - & - & $957(40.95)$ & \\
\hline Infliximab & - & - & $419(17.93)$ & \\
\hline Adalimumab & - & - & $934(39.97)$ & \\
\hline Golimumab & - & - & $27(1.16)$ & \\
\hline
\end{tabular}

Values are presented as mean \pm SD or number (\%).

csDMARD, conventional synthetic disease-modifying anti-rheumatic drug; TNFi, tumor necrosis factor inhibitors; MTX, methotrexate. 
tients with and without anti-TNF therapy $(p=0.471)$.

\section{Cancer incidence}

Incidence rates for all cancers and common cancers are presented in Table 4. The most common cancer was thyroid cancer, followed by lung cancer, gastric cancer, colon cancer, breast cancer, and liver cancer in descending order. Lymphoma, a common cancer in RA patients, was not detected in patients treated with TNFi.

The overall cancer incidence was higher in the csDMARDs group than in the TNFi group. However, when the IRRs were adjusted for age, gender, and observational period, there was no difference in cancer incidence for any kind between patients treated with csDMARDs and those treated with TNFi.

\section{DISCUSSION}

This study demonstrated that malignancy incidence was similar in seropositive RA patients treated with TNFi and those treated with csDMARDs. The IRRs adjusted for age, gender, and observational period revealed that the incidence for all cancers and site-specific cancers did not differ significantly between the groups.

To our knowledge, this is the first study comprising all citizens to investigate the cancer incidence in RA subsequent to anti-TNF therapy. A single study conducted in Taiwan included all citizens treated with TNFi [12]. The Taiwanese study also used their National Health Insurance Database, and selected matched controls from patients treated with csDMARDs. We were able to minimize the drop-out that affects the detection

Table 2. Comparison between rheumatoid arthritis patients with and without cancer

\begin{tabular}{|c|c|c|c|}
\hline Variable & Patient without cancer $(n=43,642)$ & Patient with cancer $(n=1,781)$ & $p$ value \\
\hline Age, yr & $57.23 \pm 13.23$ & $63.71 \pm 12.07$ & $<0.001$ \\
\hline Male sex & $8,695(19 \cdot 92)$ & $516(28.97)$ & $<0.001$ \\
\hline Distribution of TNFi & & & 0.268 \\
\hline Etanercept & $941(41.13)$ & $16(32.65)$ & \\
\hline Infliximab & $412(18.01)$ & $7(14.29)$ & \\
\hline Adalimumab & $908(39.69)$ & $26(53.06)$ & \\
\hline Golimumab & $27(1.18)$ & o & \\
\hline
\end{tabular}

Values are presented as mean $\pm \mathrm{SD}$ or number (\%).

TNFi, tumor necrosis factor inhibitors.

Table 3. Cancer detection in patients treated with csDMARDs and TNFi during the study period

\begin{tabular}{|c|c|c|c|c|}
\hline Variable & All patient $(n=45,423)$ & $\begin{array}{l}\text { Patient treated with csDMARD } \\
\qquad(\mathrm{n}=43,086)\end{array}$ & $\begin{array}{l}\text { Patient treated with TNFi } \\
\qquad(\mathrm{n}=2,337)\end{array}$ & $p$ value \\
\hline Patient-years & 145,944 & 137,746 & 8,037 & $<0.001$ \\
\hline Cancer case & $1,781(3.92)$ & $1,732(4.02)$ & $49(2.10)$ & $<0.001$ \\
\hline Events/100,000 patient-years & $1,220.33$ & $1,257 \cdot 39$ & 600.26 & $<0.001$ \\
\hline $\begin{array}{l}\text { Cancer diagnosis calendar } \\
\text { year, } \mathrm{n} \text { (\% within all cancer) }\end{array}$ & & & & 0.038 \\
\hline 2010 & $378(21.22)$ & $372(21.48)$ & $6(12.24)$ & \\
\hline 2011 & $465(26.11)$ & $456(26.33)$ & $9(18.37)$ & \\
\hline 2012 & $472(26.50)$ & $459(26.50)$ & $13(26.53)$ & \\
\hline 2013 & $466(26.17)$ & $445(25.69)$ & $21(42.86)$ & \\
\hline Time to cancer, mon & $20.06(8.56-31.56)$ & $20.21(8.66-31.77)$ & $18.60(7.58-29.62)$ & 0.471 \\
\hline
\end{tabular}

Values are presented as number (\%) or median (interquartile range).

csDMARD, conventional synthetic disease-modifying anti-rheumatic drug; TNFi, tumor necrosis factor inhibitors. 
Table 4. Cancer incidence in rheumatoid arthritis patients treated with csDMARDs and TNFi

\begin{tabular}{|c|c|c|c|c|c|c|c|c|}
\hline \multirow{2}{*}{ Variable } & \multicolumn{2}{|c|}{$\operatorname{Total}(\mathrm{n}=45,423)$} & \multicolumn{2}{|c|}{$\operatorname{csDMARD}(\mathrm{n}=43,086)$} & \multicolumn{2}{|c|}{ TNFi $(\mathrm{n}=2,339)$} & \multirow{2}{*}{$\frac{\text { IRR }(95 \% \text { CI })^{\mathrm{a}}}{\text { TNFi vs. csDMARDs }}$} & \multirow{2}{*}{$p$ value } \\
\hline & Case & Incidence $^{\mathrm{b}}$ & Case & Incidence $^{\mathrm{b}}$ & Case & Incidence $^{\mathrm{b}}$ & & \\
\hline All cancer & 1,781 & $1,220.33$ & 1,732 & $1,257 \cdot 39$ & 49 & $599 \cdot 57$ & $0.913(0.679-1.227)$ & 0.546 \\
\hline Thyroid cancer & 312 & $404 \cdot 52$ & 302 & 415.98 & 10 & $205 \cdot 94$ & $0.966(0.510-1.830)$ & 0.916 \\
\hline Lung cancer & 239 & 299.13 & 232 & 306.81 & 7 & 156.44 & $1.038(0.484-2.227)$ & 0.923 \\
\hline Gastric cancer & 194 & $249 \cdot 33$ & 188 & 254.57 & 6 & 151.25 & $1.146(0.503-2.610)$ & 0.745 \\
\hline Colon cancer & 180 & 234.90 & 177 & 243.80 & 3 & 70.24 & $0.623(0.198-1.965)$ & 0.419 \\
\hline Breast cancer (in female) & 147 & $199 \cdot 52$ & 142 & 202.44 & 5 & 146.92 & $1.184(0.480-2.919)$ & 0.714 \\
\hline Prostate cancer (in male) & 81 & 158.09 & 80 & 119.25 & 1 & $13 \cdot 54$ & $0.445(0.061-3.246)$ & 0.425 \\
\hline Liver cancer & 99 & 132.33 & 98 & 138.52 & 1 & 20.20 & $0.339(0.047-2.445)$ & 0.283 \\
\hline Lymphoma & 67 & 76.39 & 67 & 80.53 & o & 0.00 & - & - \\
\hline
\end{tabular}

csDMARD, conventional synthetic disease-modifying anti-rheumatic drug; TNFi, tumor necrosis factor inhibitors; IRR, incidence rate ratio; CI, confidence interval.

${ }^{a}$ Adjusted for age, gender, and observational period.

bDefined as 'cancer cases/100,000 patient-years.'

of cancer occurrence by using the HIRA database, because all citizens were registered in the HIRA database. Inclusion of all citizens requires proper selection of study population. Previously, Cho et al. [16] suggested that selecting patients according to diagnosis codes and prescribed drugs from the HIRA database was a reliable way to identify patients with RA. However, we could include only patients with seropositive RA, because of the reliability of diagnosis codes.

Previous studies have reported a wide range of cancer incidence rates, between 3.8 and 9.3 per 1,000 patient-years [10-12,17,18]. Estimation of cancer risk can vary due to ethnic diversity and environmental contributions [13]. Excluding thyroid cancer, the crude cancer incidence in Korean RA patient was 8.2/1,000 patient-years. Because we included the diagnosis of carcinoma in situ, the estimated incidence would be relatively higher compared to the previous research. In a prior study conducted in Korea between 2001 and 2007, the overall cancer incidence in RA patients was 4.6/1,000 patient-years [19]. This cancer occurrence could have been underestimated, because enrollment and follow-up for RA patients was limited to a single center. Nonetheless, the incidence was much lower than that identified in this study. This discrepancy may be due to differences in patient characteristics and study periods. The present study included a higher proportion of older and male patients, who develop cancers more frequent- ly, according to the National Cancer Information Center [20]. Moreover, cancer incidence has increased continuously in the general population since 2000 , and the same trend should be expected in RA patients.

Previously, clinical studies investigating the association between anti-TNF therapy and cancer have shown inconsistent results. An initial-stage meta-analysis of clinical trials revealed increased cancer incidence in patients treated with adalimumab and infliximab [8]. However, the observational period, ranging from 3 months to 1 year, was too short to evaluate the carcinogenic effects of TNFi. Subsequent observational studies with larger sample sizes and longer durations revealed similar cancer incidences, although there were controversies regarding several kinds of cancers [21]. Recently, a Taiwanese nationwide cohort study demonstrated that cancer risk was lower in patients treated with anti-TNF therapy [12]. The overall cancer incidence excluding hematologic malignancy and standardized incidence ratios for lung, breast, and genitourinary cancers were significantly lower in the biologics cohort [12]. Our study also indicated that crude cancer incidence was lower in patients treated with TNFi than in those treated with csDMARDs. However, there were significant differences between the groups in clinical characteristics affecting cancer incidence, such as age, gender, and follow-up duration. When the IRRs were adjusted for age, gender, and observational period, incidence rates for all cancers 
and site-specific cancers were comparable in patients treated with csDMARDs and TNFi.

The pathogenesis of cancer is closely linked to inflammation and immunity [22]. In general, chronic inflammation triggered by infection or autoimmunity can be a risk factor for cancer occurrence. In RA patients, longstanding disease and high disease activity were found to increase cancer risk, probably through chronic inflammatory response $[23,24]$. Thus, the effective control of inflammation by TNFi might reduce the cancer risk, although the effects of TNF on carcinogenesis are controversial [5]. However, to elucidate causal links between anti-TNF therapy and cancer, further research will be required.

The most common cancer in all patients with RA was thyroid cancer, followed by lung cancer, stomach cancer, and colon cancer in descending order. The high incidence of thyroid cancer is in line with its incidence in the general population during the study period, due to increased medical surveillance [25]. Except thyroid cancer, the most common site of cancer was lung in RA patients, whereas stomach cancer was most common in the general population during the study period. An increased risk for lung cancer in RA patients has been suggested by many previous studies [26]. In this study, lymphoma was not detected among TNFi-treated patients.

The present study has several limitations. First of all, it was difficult to analyze the effects of other confounding variables, such as comorbidities, medications, and disease activity and duration. Information about comorbidities and medications was insufficient for further analysis due to the limitations of the database. Moreover, disease-specific features were not accessible, because of personal information protection. However, we assumed that the csDMARDs group also had active RA requiring methotrexate, leflunomide, or combination of csDMARDs. Second, patients with seronegative RA were not included in the analysis. Third, the study period was 4 years, and the mean follow-up duration was 3.2 years, which is a relatively short period for evaluating TNFi effects on carcinogenesis. Finally, we did not compare cancer incidence between RA patients and the general population, due to limited HIRA database information and cancer statistics.

This study showed the nationwide cancer incidence in Korean patients with RA and evaluated the associa- tion between anti-TNF therapy and cancer risk, using the database of all citizens. However, we should be aware that this study provides only rudimentary information, which requires further evaluation using well-designed registry or cohort. Recently, the Korean College of Rheumatology has constructed a national biologics registry for safety evaluation in treatment with biologics [27]. Forthcoming data from this nationwide registry will provide a better understanding of the association between biologic therapies and cancer.

In conclusion, the risk of malignancy did not differ significantly between RA patients treated with TNFi and those treated with csDMARDs based on the insurance claim data. In terms of malignancy, anti-TNF therapy may be an effective therapeutic option with an acceptable safety profile for patients with inadequate responses to csDMARDs. This will be illuminated more thoroughly in a prospective observational registry set up.

\section{KEY MESSAGE}

1. We used the data from National Health Insurance claims database to determine the risk of cancer in patients with rheumatoid arthritis (RA).

2. The risk of malignancy did not differ significantly between patients treated with tumor necrosis factor (TNF) inhibitors and conventional synthetic disease modifying anti-rheumatic drugs.

3. Anti-TNF therapy would be a safe therapeutic option for active RA, in terms of malignancy.

\section{Conflict of interest}

No potential conflict of interest relevant to this article was reported.

\section{Acknowledgments}

This study was supported by a 2013-Grant from Korean Academy of Medical Sciences. The funding source had no involvement in the study design, collection, analysis, and interpretation of data; in the writing of the manuscript; or in the decision to submit the manuscript for publication. 


\section{REFERENCES}

1. Singh JA, Saag KG, Bridges SL Jr, et al. 2015 American College of Rheumatology Guideline for the treatment of rheumatoid arthritis. Arthritis Rheumatol 2016;68:1-26.

2. Smolen JS, Landewe R, Breedveld FC, et al. EULAR recommendations for the management of rheumatoid arthritis with synthetic and biological disease-modifying antirheumatic drugs: 2013 update. Ann Rheum Dis 2014; 73:492-509.

3. Nam JL, Ramiro S, Gaujoux-Viala C, et al. Efficacy of biological disease-modifying antirheumatic drugs: a systematic literature review informing the 2013 update of the EULAR recommendations for the management of rheumatoid arthritis. Ann Rheum Dis 2014;73:516-528.

4. Lebrec H, Ponce R, Preston BD, Iles J, Born TL, Hooper M. Tumor necrosis factor, tumor necrosis factor inhibition, and cancer risk. Curr Med Res Opin 2015;31:557-574.

5. Balkwill F. Tumour necrosis factor and cancer. Nat Rev Cancer 2009;9:361-371.

6. Green S, Dobrjansky A, Carswell EA, et al. Partial purification of a serum factor that causes necrosis of tumors. Proc Natl Acad Sci U S A 1976;73:381-385.

7. Carswell EA, Old LJ, Kassel RL, Green S, Fiore N, Williamson B. An endotoxin-induced serum factor that causes necrosis of tumors. Proc Natl Acad Sci U S A 1975;72:36663670 .

8. Bongartz T, Sutton AJ, Sweeting MJ, Buchan I, Matteson EL, Montori V. Anti-TNF antibody therapy in rheumatoid arthritis and the risk of serious infections and malignancies: systematic review and meta-analysis of rare harmful effects in randomized controlled trials. JAMA 2006;295:2275-2285.

9. Lopez-Olivo MA, Tayar JH, Martinez-Lopez JA, et al. Risk of malignancies in patients with rheumatoid arthritis treated with biologic therapy: a meta-analysis. JAMA 2012; 308:898-908.

10. Mercer LK, Lunt M, Low AL, et al. Risk of solid cancer in patients exposed to anti-tumour necrosis factor therapy: results from the British Society for Rheumatology Biologics Register for Rheumatoid Arthritis. Ann Rheum Dis 2015;74:1087-1093.

11. Buchbinder R, Van Doornum S, Staples M, Lassere M, March L. Malignancy risk in Australian rheumatoid arthritis patients treated with anti-tumour necrosis factor therapy: analysis of the Australian Rheumatology Asso- ciation Database (ARAD) prospective cohort study. BMC Musculoskelet Disord 2015;16:309.

12. Wu CY, Chen DY, Shen JL, et al. The risk of cancer in patients with rheumatoid arthritis taking tumor necrosis factor antagonists: a nationwide cohort study. Arthritis Res Ther 2014;16:449.

13. Torre LA, Bray F, Siegel RL, Ferlay J, Lortet-Tieulent J, Jemal A. Global cancer statistics, 2012. CA Cancer J Clin 2015;65:87-108.

14. National Health Insurance Service. Operational structure of Korea health care system [Internet]. Wonju (KR): National Health Insurance Service, c2013 [cited 2017 Sep 21]. Available from: http://www.nhis.or.kr/static/html/wbd/g/ a/wbdgao401.html.

15. Jung SM, Ju JH, Park MS, et al. Risk of tuberculosis in patients treated with anti-tumor necrosis factor therapy: a nationwide study in South Korea, a country with an intermediate tuberculosis burden. Int J Rheum Dis 2015; 18:323-330.

16. Cho SK, Sung YK, Choi CB, Kwon JM, Lee EK, Bae SC. Development of an algorithm for identifying rheumatoid arthritis in the Korean National Health Insurance claims database. Rheumatol Int 2013;33:2985-2992.

17. Mariette X, Matucci-Cerinic M, Pavelka K, et al. Malignancies associated with tumour necrosis factor inhibitors in registries and prospective observational studies: a systematic review and meta-analysis. Ann Rheum Dis 2011;70:1895-1904.

18. Chen YJ, Chang YT, Wang CB, Wu CY. The risk of cancer in patients with rheumatoid arthritis: a nationwide cohort study in Taiwan. Arthritis Rheum 2011;63:352-358.

19. Kim YJ, Shim JS, Choi CB, Bae SC. Mortality and incidence of malignancy in Korean patients with rheumatoid arthritis. J Rheumatol 2012;39:226-232.

20. National Cancer Information Center. Cancer incidence [Internet]. Goyang (KR): National Cancer Information Center, c2012 [cited 2017 Sep 21]. Available from: http://www.cancer. go.kr/mbs/cancer/subview.jsp?id=cancer_040101000000.

21. Chen Y, Sun J, Yang Y, Huang Y, Liu G. Malignancy risk of anti-tumor necrosis factor alpha blockers: an overview of systematic reviews and meta-analyses. Clin Rheumatol 2016;35:1-18.

22. Grivennikov SI, Greten FR, Karin M. Immunity, inflammation, and cancer. Cell 2010;140:883-899.

23. Abasolo L, Judez E, Descalzo MA, et al. Cancer in rheumatoid arthritis: occurrence, mortality, and associated 
factors in a South European population. Semin Arthritis Rheum 2008;37:388-397.

24. Baecklund E, Iliadou A, Askling J, et al. Association of chronic inflammation, not its treatment, with increased lymphoma risk in rheumatoid arthritis. Arthritis Rheum 2006;54:692-701.

25. Oh CM, Jung KW, Won YJ, Shin A, Kong HJ, Lee JS. Age-period-cohort analysis of thyroid cancer incidence in Korea. Cancer Res Treat 2015;47:362-369.
26. Simon TA, Thompson A, Gandhi KK, Hochberg MC, Suissa S. Incidence of malignancy in adult patients with rheumatoid arthritis: a meta-analysis. Arthritis Res Ther 2015;17:212.

27. Korean College of Rheumatology. Korean College of Rheumatology Biologics Registry [Internet]. Seoul (KR): Korean College of Rheumatology, c2006 [cited 2017 Sep 21]. Available from: http://www.rheum.or.kr/kobio/. 